\title{
Implementation of wheelchair controller using mouth and tongue gesture
}

\author{
Rafia Hassani, Mohamed Boumehraz, Maroua Hamzi \\ Department of Electrical Engineer, Faculty of sciences and Technology, University of Biskra, Aljazair, Algeria
}

\begin{tabular}{l}
\hline \hline Article Info \\
\hline Article history: \\
Received Jun 5, 2021 \\
Revised Oct 19, 2021 \\
Accepted Oct 25, 2021 \\
\hline
\end{tabular}

\section{Keywords:}

Face detection

Human machine interface

Mouth gesture

Powered wheelchair

Tongue gesture

\begin{abstract}
In this paper, a simple human-machine interface allowing people with severe disabilities to control a motorized wheelchair using mouth and tongue gesture is presented. The development of the proposed system consists of three principal phases: the first phase is mouth detection which performed by using haar cascade to detect the face area and template matching to detect mouth and tongue gestures from the lower face region. The second phase is command extraction; it is carried by determining the mouth and tongue gesture commands according to the detected gesture, the time taken to execute the gestures, and the previous command which is stored in each frame. Finally, the gesture commands are sent to the wheelchair as instruction using the Bluetooth serial port. The hardware used for this project were; laptop with universal serial bus (USB) webcam as a vision-based control unit, Bluetooth module to receive instructions comes from the vision control unit, standard joystick used in case of emergency, joystick emulator which delivers to the control board signals similar to the signals that are usually generated by the standard joystick, and ultrasonic sensors to provide safe navigation. The experimental results showed the success of the proposed control system based on mouth and tongue gestures.
\end{abstract}

This is an open access article under the CC BY-SA license.

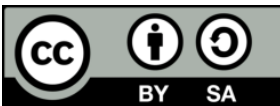

\section{Corresponding Author:}

Rafia Hassani

Department of Electrical Engineer, Faculty of sciences and Technology

University of Biskra, Aljazair, Algeria

Email: rafia.hassani@univ-biskra.dz

\section{INTRODUCTION}

With the world's population aging and the number of handicapped persons due to diseases, wars, and accidents mounting; there is a growing demand from international health care organization, laboratories, and companies interested in innovating new technologies that can help this group of people. Wheelchairs are important devices of transport for this population. However, traditional wheelchairs controlled by users via joysticks, buttons, and levers cannot satisfy the requirements of disabled users suffering from quadriplegia or amputee's hands, or of paralyzed patients who have lost the ability to use their arms. This segment of people needs to use a special control system depending on their abilities. In order to find a solution to this problem, various methods and techniques have been developed to create intelligent wheelchairs (IWs) over the last years [1], [2]. According to the general concept presented in the relevant literature, an IW is defined as a standard powered wheelchair equipped with sensors and a computer, allowing it to have certain intelligence.

Due to the extensive availability and low cost of commercial voice recognition hardware, speech recognition has often been used for controlling IWs. The methods presented in [3], [4] use the speech to control the wheelchair movement. Aruna et al. [5], the speech recognition technique is used to switch 
between modes. Speech-based wheelchair control is very efficient and accurate. However, the voice recognition rate may decrease when the surrounding environment is noisy.

Bio-signals-based methods like electromyography (EMG) [6], [7], electro-oculography (EOG) [8]-[10], and electro-encephalography (EEG) [11], [12], are used for completely paralyzed patients who can only use their bio-signal as a resource to control their wheelchairs. In these methods, the patient can easily command the wheelchair by his/her bio-signal. Unfortunately, the accuracy of these approaches is still low, and problems may arise while trying to use a bio-signal based human-machine-interface (HMI) because they vary greatly between individuals. Also, in the same person, they change substantially over time.

Hassani et al. [13], gyro-accelerometer-based method is presented. The wheelchair is controlled using head tilt. Firstly, the user inclines his head in left/right/front or back direction. Then, the acceleration and rotation rate of the movement are detected as a raw value by using the accelerometer and the gyroscope sensors. This data is sent to a microcontroller for processing and calculating the proper pitch and roll angles of user head inclination using the complex geometry and Kalman filter for sensor fusion. Depending on the calculated angles, the microcontroller controls the wheelchair. But this method is not comfortable for handicapped people and may have caused them some illnesses in their neck.

Vision-based methods are another trend in control interfaces. The authors in [14], [15] have used the eye-tracking method as a computer vision interface to allow paralyzed users to drive by eye. However, the eye movements which used to control the wheelchair may force and affect the user's vision, causing tiredness and dizziness.

Some human head gesture-based interfaces were used to control a wheelchair [16]-[19]. Hassani et al. [16], haar cascade and mean shift algorithm is used to detect user's face, to track it and then to recognize user gesture. After the head gesture is estimated, it used to control the direction of the electric wheelchair. Where, while, the head gesture recognition algorithm presented in [17] uses the position of the user's lips compared with a fixed rectangular window to define the head movement. Qassim et al. [18], a webcam was set up in front of the user to acquire a real-time video of a user's face and to detect its position in the whole image, then the face position is translated to required controlling signals, these latest are generated and sent to the microcontroller via universal serial bus (USB) port to control the wheelchair. The system proposed in [19] allows the user to move the electric wheelchair using their face tilting and their mouth shape information. So that if the user wants to turn right or left, they need to incline their face to the right or to the left, respectively. If they want to go forward or backward then, they need to open or to close their mouth. However, it is difficult to control electric wheelchair by head or face movement.

Also, hand gesture recognition is used as a method for controlling an electric wheelchair. Rabhi et al. [20] used visual recognition algorithm and artificial intelligence software to recognize the patient's hand movements; the derived corresponding signals are thus used to control the electric wheelchair in real time. However, this method is not considered solutions to multiple sclerosis patients, quadriplegic, and amputee's hand patients.

In this work, the implementation of wheelchair controller using mouth and tongue gestures is presented. The system comprises two parts: the vision-based control unit, which represents the user interface and uses simple gestures allowing people with severe disabilities to guide the wheelchair. Meanwhile, the second part represents the base control unit which comprises an electric wheelchair equipped with necessary electronics (a Bluetooth module, ultrasonic sensors, and Arduino Mega). The complete details of this system are explained in research method section 2. The experimental results achieved by our system are presented in section 3. Finally, section 4 presents a brief conclusion and perspective.

\section{RESEARCH METHOD}

\subsection{System design}

Figure 1 shows the design of our proposed system. It based on a commercial electric wheelchair equipped with the joystick. Our system consists of:

- Vision-based control unit: contains the USB camera and laptop, which are used to process user input and sends the control commands to the base control unit.

- Base control unit: comprises three ultrasonic sensors placed at the bottom of the wheelchair to provide safe navigation, a Bluetooth module to receive command instruction comes from the vision control unit, a standard joystick used in manual mode or in case of emergencies, a microcontroller card composed of an Arduino Mega and an operational amplifier based low-pass filter used to emulate the joystick.

One of the main advantages of this concept is the automatic switching between the manual mode and the visual mode. If the joystick is active, then the joystick commands are used to control the wheelchair and the visual mode is inhibited. Once the joystick is inactive, the commands delivered by the mouth gesture mode are used to control the wheelchair while keeping the priority to the manual mode. Another advantage is 
that users can dispense with the vision-based control unit if they can control the wheelchair using the standard joystick without changing the base control unit.

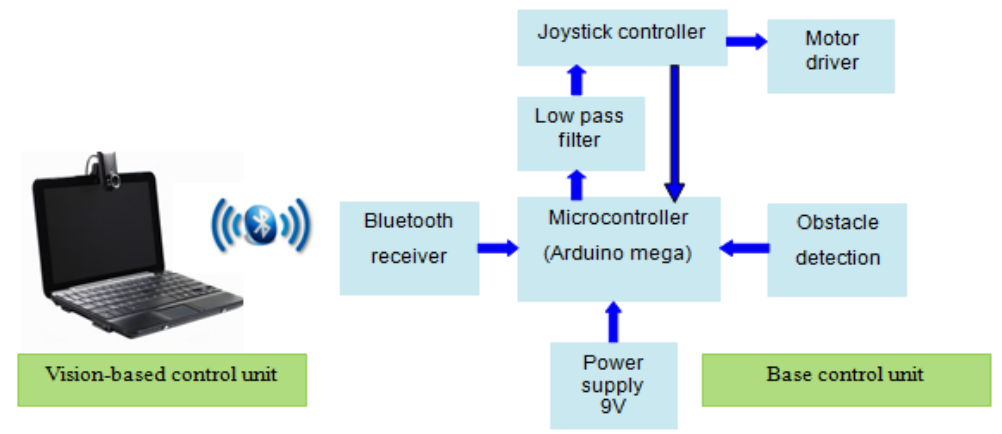

Figure 1. System design

\subsection{Vision interface}

This interface allows the user with severe physical disabilities to guide the wheelchair using few and simple gestures (mouth and tongue gestures). After image acquisition from the camera, the user face area is detected using a haar cascade classifier to extract the approximate mouth region. For our project, OpenCV [21] is used to implement the haar cascade classifier [22], [23]. Haar cascade classifier is a machine learning-based approach where a cascade function is trained from a lot of positive images (which contain the object that wants to detect it) and negative images (which do not contain the object that wants to detect it). Subsequently, it is used to detect objects in other images. Figure 2 shows the flowchart of mouth and tongue gestures control method. After the system determines the approximate mouth region, template matching is performed for detecting the mouth. Template matching is a simple technique for finding areas of an image that match a template image [24]. Before performing template matching, we load three template images to the system (shown in Figure 2). These images present the motions that can be used by the mouth for controlling the wheelchair movement.

To apply the template matching technique, matchTemplate function which built into OpenCV is used. This function requires three parameters. The first is the input source image I, the image that contains what we are searching for. Here, we selected the lower face image as the input source image. The second is template image $\mathrm{T}$; this image is contained within the input source image and we are looking to select its location. In our system, we used three template images which mean that we applied matchTemplate function three times, one for each template image in the frame. Finally, the third parameter is our template matching method, there are varieties of methods available in OpenCV, but in our system, we used the correlation coefficient which is specified by the flag CV_TM_CCOEFF_NORMED. The equation for this method is presented as (1) [24].

$$
R(x, y)=\frac{\sum_{x^{\prime}, y^{\prime}}\left[\mathrm{T}^{\prime}\left(\mathrm{x}^{\prime}, \mathrm{y}^{\prime}\right) \cdot \mathrm{I}^{\prime}\left(\mathrm{x}+\mathrm{x}^{\prime}, \mathrm{y}+\mathrm{y}^{\prime}\right)\right]}{\sqrt{\sum_{\mathrm{x}^{\prime}, \mathrm{y}^{\prime}}\left[\mathrm{T}^{\prime}\left(\mathrm{x}^{\prime}, \mathrm{y}^{\prime}\right)^{2} \cdot \mathrm{I}^{\prime}\left(\mathrm{x}+\mathrm{x}^{\prime}, \mathrm{y}+\mathrm{y}^{\prime}\right)^{2}\right]}}
$$

Where $\mathrm{R}$ is numerical index for correlation coefficient, $\mathrm{T}^{\prime}$ represents the average value of template $\mathrm{T}$, $I^{\prime}$ represents the average value of source image $I$ in the region coincide with template $T, x^{\prime}=$ $0 \ldots \ldots \ldots$ width -1 and $\mathrm{y}^{\prime}=0 \ldots \ldots$ high -1 .

Once the match was found in the source image, we excluded a rectangle around the area corresponding to the highest match. Then, according to which gesture from the three template images is detected (open mouth, tongue on the right, or tongue on the left), the time taken to execute the gesture, and the previous command stored in each frame, the gesture command is determined and sent to the serial port as instruction. Five commands control the wheelchairs, and two other commands are used to stop and start this system (Table 1).

- If the user takes out his/her tongue on the left, the gesture is recognized as the left turn command.

- If the user takes out his/her tongue on the left, the gesture is recognized as the right turn command.

- If the user opens his mouth, the system triggers a timer to calculate the time taken before closing his/her mouth $(\mathrm{t})$.

- If $t \leq 2 \mathrm{~s}$ and previous command is "left, right, forward or backward", the gesture is recognized as stop command. 
- If $t \leq 2 \mathrm{~s}$ and previous command is "stop", the gesture is recognized as forward command.

- If $2 s<t \leq 7 s$ and previous command is "stop", the gesture is recognized as backward command.

- If $t>7 \mathrm{~s}$ and previous command is "left, right, stop, forward or backward", mouth gesture recognition controller will be stopped.

- If $t>7 \mathrm{~s}$ and previous command is "stop controlling", mouth gesture recognition controller will be started.

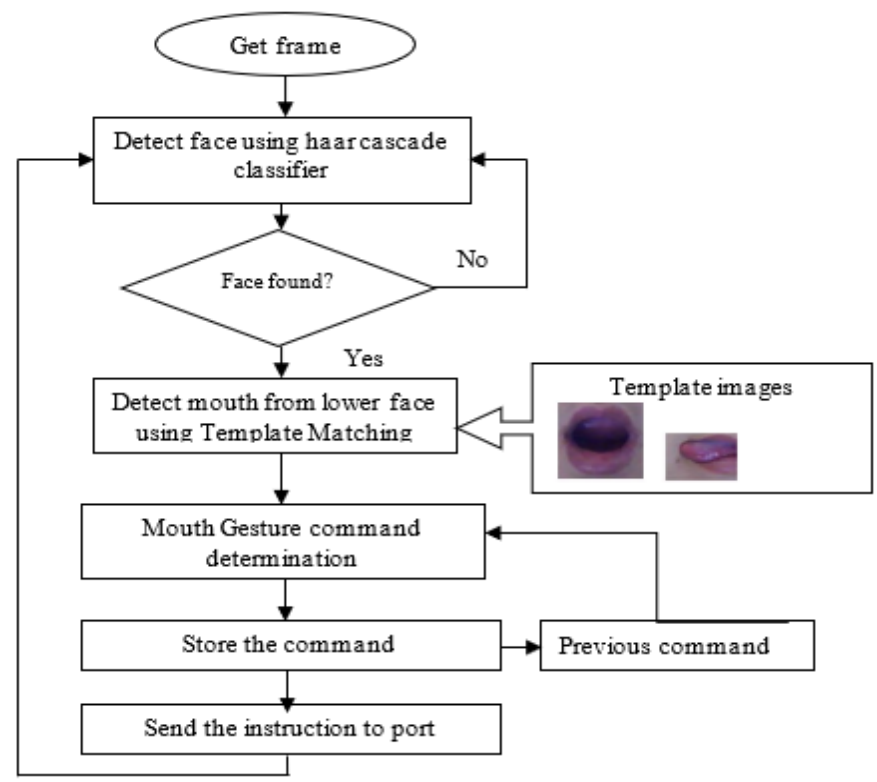

Figure 2. The flowchart of mouth and tongue gestures control method

Table 1. Mouth gesture used to control the wheelchair

\begin{tabular}{lll} 
Mouth and tongue gestures & Time $(\mathrm{s})$ & \multicolumn{1}{c}{ Previous command } \\
& $/$ & Left, right, stop, forward or backward \\
& & Left, right, stop, forward or backwar \\
$2 \mathrm{~s}<\mathrm{t} \leq 7 \mathrm{~s} \quad$ Left, right, stop, forward or backward \\
$\mathrm{t}>7 \mathrm{~s} \quad$ Left, right, stop, forward or backward \\
Stop controlling
\end{tabular}

\subsection{User interface}

The system uses Microsoft visual C\#, and arduino-1.0 as a software program environment. Image and video acquisition and processing, face and mouth detection are achieved using OpenCV library functions. Figure 3 shows the user interface, the left side is windows showing real-time image from the 
camera, capture/stop button used to start or to stop capturing the image in real-time, connexion button used to send the instruction to the wheelchair via Bluetooth serial port, time label presents the time taken when executing open mouth motion, and command label shows the mouth gesture recognition result.

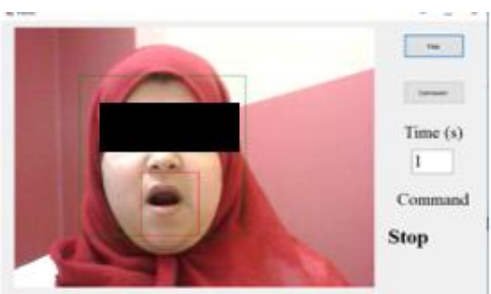

Figure 3. The user interface

\subsection{Base control unit}

To integrate the proposed mouth and tongue gestures control method to the wheelchair, a small change was made to its control box by adding a Bluetooth transmission module to receive instructions from the vision-based control unit and safety sensors to stop the wheelchair in case of an obstacle, and designing a virtual joystick using an Arduino, which delivers to the wheelchair the same signals coming from $\mathrm{X}$ and $\mathrm{Y}$ axis of the joystick if the joystick is active and emulates joystick signals according to command instructions from the vision-based control unit if the joystick is inactive. The voltage ranges of the two outputs generated from the $\mathrm{X}$ and $\mathrm{Y}$-axis movement of the joystick, which are used to guide the wheelchair, are presented in Table 2 (JC 2000 contactless joystick from Curtiss-Wright) [25]. The joystick movement along the X-axis makes the wheelchair turn right or left, and its movement along the Y-axis makes the wheelchair go forward or backward. To make the microcontroller board emulate joystick signals, its pulse width modulation (PWM) outputs are used [26]. Then, the PWM outputs are converted into voltage signals using a low-pass filter. In order to get analog voltage signals with a minimal ripple and a fast response, a 2nd-order RC low-pass filter was implemented with the following component value: $\mathrm{R} 1=200 \Omega$. R1=R2=200 $\Omega, \mathrm{C} 1=\mathrm{C} 2=22 \mu \mathrm{F}$. Figure 4 shows the base control unit circuit.

Table 2. The joystick voltage ranges

\begin{tabular}{lcc}
\hline & V1(V) & V2 (V) \\
\hline Stop & 2.5 & 2.5 \\
Right & $2.5-3.9$ & 2.5 \\
Left & $1.1-2.5$ & 2.5 \\
Forward & 2.5 & $2.5-3.9$ \\
Backward & 2.5 & $1.1-2.5$ \\
\hline
\end{tabular}

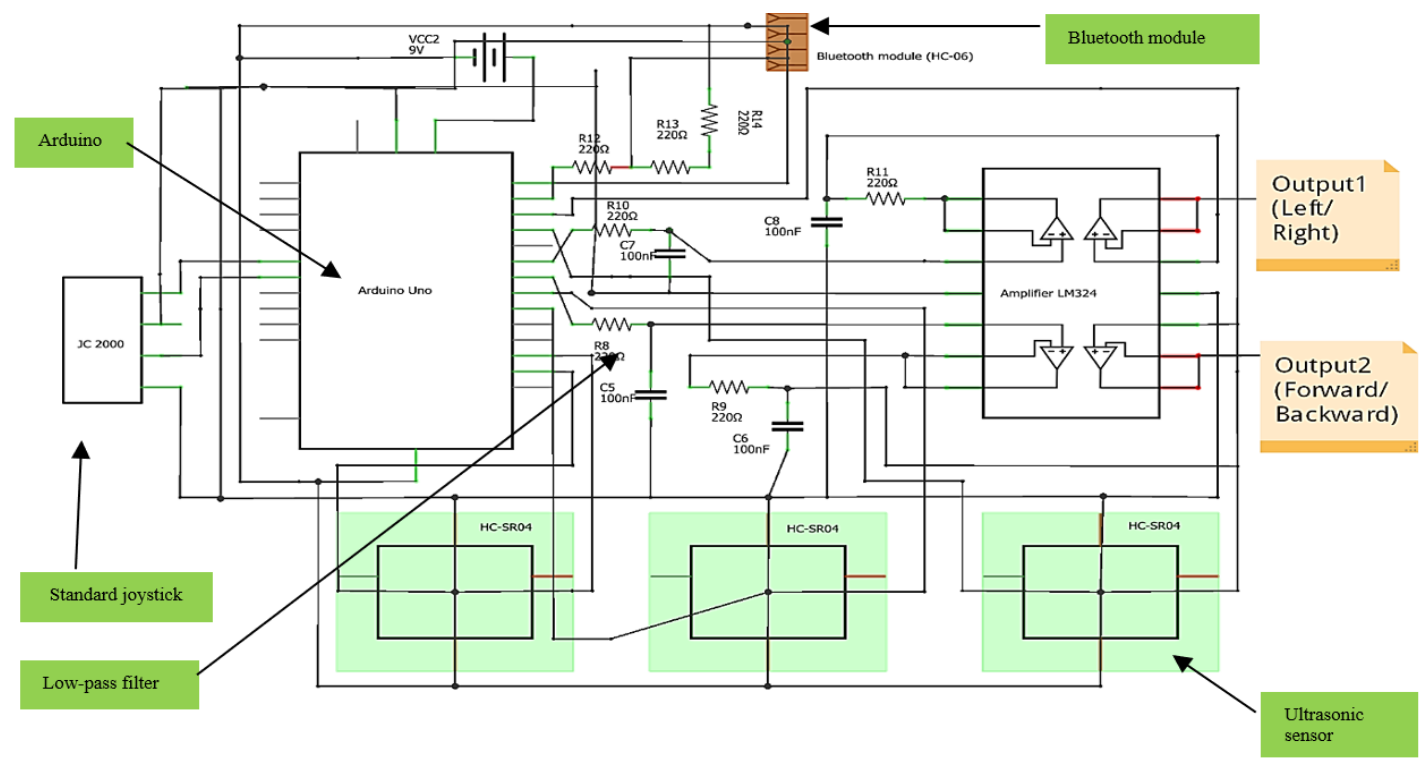

Figure 4. Base control unit circuit 


\section{RESULTS AND DISCUSSION}

\subsection{Recognition experiment}

In order to test the accuracy and the effectiveness of our proposed interface, two persons (male and female) were asked to apply the commands twenty times in scenes that had a complex background with three different light conditions (dark, normal, and bright). The results of recognition experiments are summarized in Table 3, and some mouth gesture recognition results in a dark, normal and bright light condition is shown in Figure 5. As indicated in the Figure 5(a)-(c), the detected face is drawn in green color, and the recognized mouth gesture result is drawn in red color and written in command Label which is located in the right corner of the interface, the results shows that the system accurately recognized the mouth gesture. This confirms its robustness to time-varying illumination and its low sensitivity to a complex environment. Figure 6 shows the three different voltages generated by the base control unit circuit when applying the commands comes from vision-based control unit (a) output voltage $(3.56 \mathrm{~V})$, (b) output voltage $(1.74 \mathrm{~V})$, and (c) output voltage $(2.48 \mathrm{~V})$. The output voltages generated by the base control unit for the five commands are:

- Output1=2.48 V and Output2=3.56 V for forward movement.

- Output1=2.48 V and Output2=1.74 V for backward movement.

- Output1=3.56 V and Output2=2.48 V for a right turn.

- Output1=1.74 V and Output2=2.48 V for a left turn.

- Output $1=2.48 \mathrm{~V}$ and Output2=2.48 V for stopping.
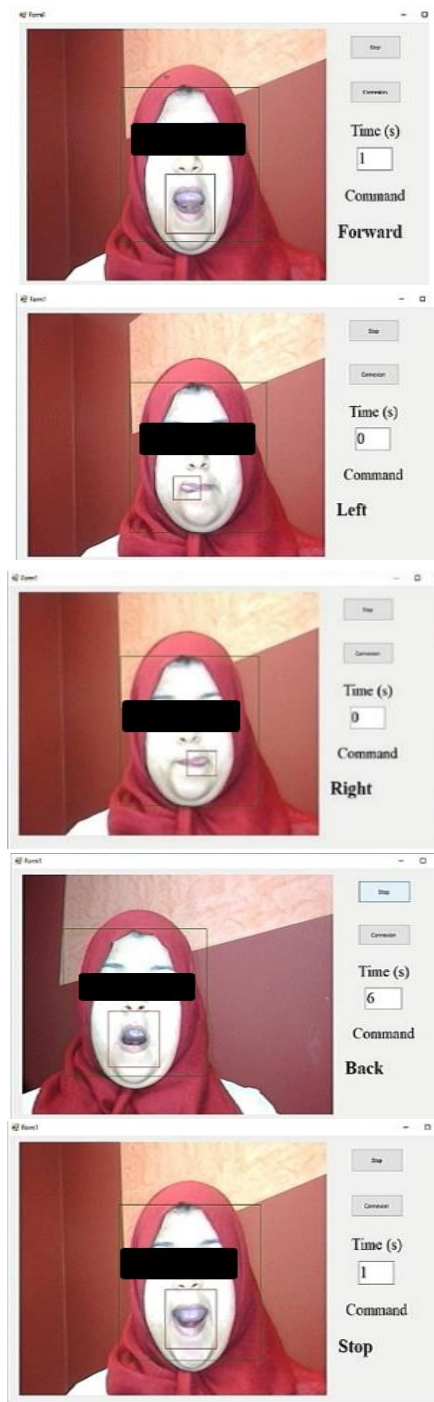

(a)
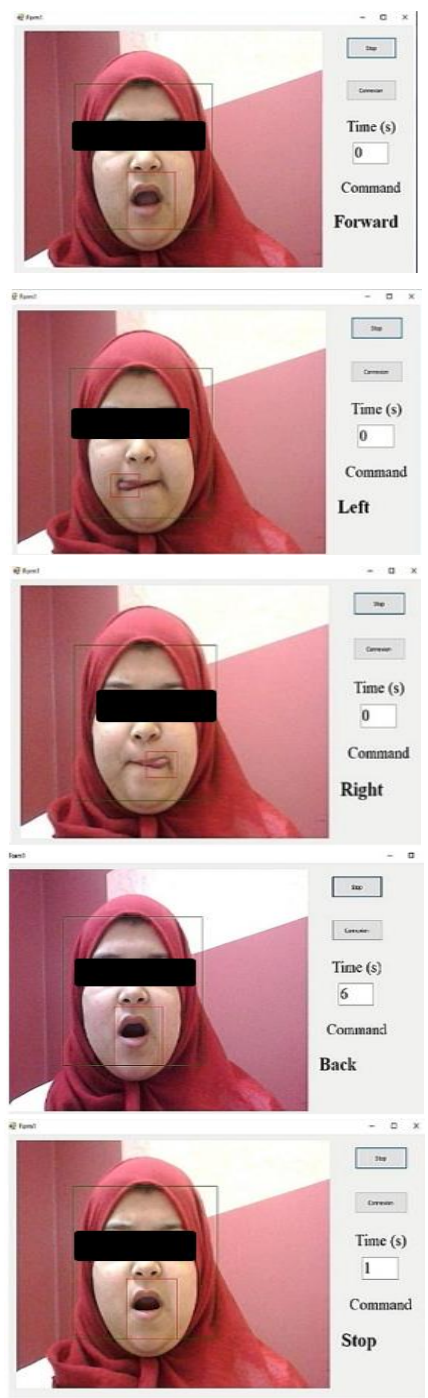

(b)
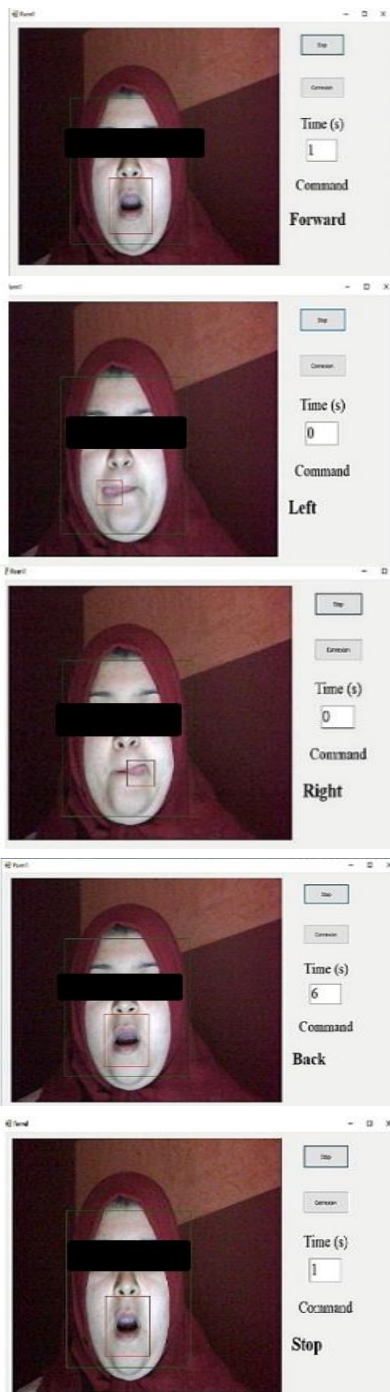

(c)

Figure 5. Mouth and tongue gestures recognition results: (a) bright light, (b) normal light, and (c) dark light 
Table 3. The results of the system for both users

\begin{tabular}{|c|c|c|c|c|c|c|}
\hline \multirow[b]{2}{*}{ Command } & \multicolumn{2}{|c|}{ Bright light condition } & \multicolumn{2}{|c|}{ Normal light condition } & \multicolumn{2}{|c|}{ Dark light condition } \\
\hline & User 1 & User 2 & User 1 & User 2 & User 1 & User 2 \\
\hline Forward & 20 & 19 & 20 & 20 & 19 & 19 \\
\hline Backward & 19 & 19 & 19 & 20 & 19 & 19 \\
\hline Left & 19 & 18 & 18 & 19 & 19 & 18 \\
\hline Right & 19 & 19 & 19 & 19 & 19 & 18 \\
\hline Stop & 20 & 20 & 20 & 20 & 20 & 20 \\
\hline Total & \multicolumn{2}{|c|}{192} & \multicolumn{2}{|c|}{194} & \multicolumn{2}{|c|}{190} \\
\hline Accuracy & \multicolumn{2}{|c|}{$96 \%$} & \multicolumn{2}{|c|}{$97 \%$} & \multicolumn{2}{|c|}{$95 \%$} \\
\hline
\end{tabular}

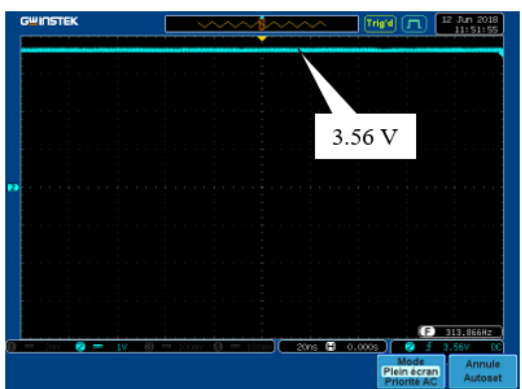

(a)

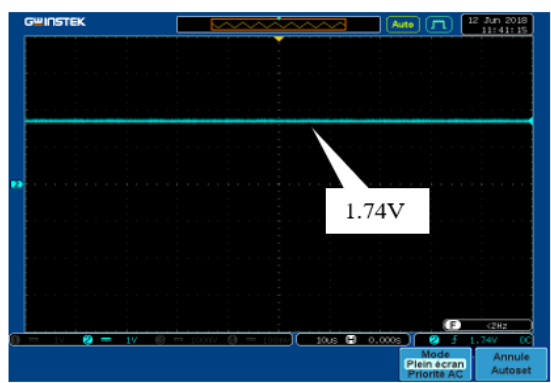

(b)

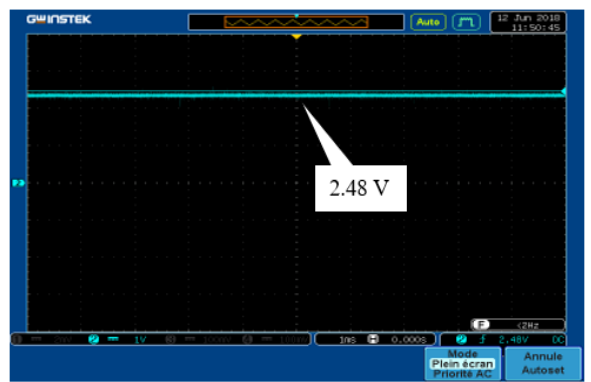

(c)

Figure 6. The three different voltages generated from the base control unit circuit:

(a) output voltage $(3.56 \mathrm{~V}),(\mathrm{b})$ output voltage $(1.74 \mathrm{~V})$, and (c) output voltage $(2.48 \mathrm{~V})$

\subsection{Running experiment in a laboratory environment}

Both the control mode of the proposed system, mouth gesture mode and manual mode were tested by controlling the wheelchair in a laboratory environment with a healthy person. In order to apply the five commands (forward, backward, right, left, and stop), we choose to follow the route presented in Figure 7. As can be seen, the width of the wheelchair is about $60 \mathrm{~cm}$; the distance between disks is $2 \mathrm{~m}$, and the total distance is about $9 \mathrm{~m}$. Three experiments were conducted to evaluate the performance of each mode. In each experiment, we recorded the traveling time and we calculate the number of input commands used to navigate along the route. The results are summarized in Table 4 and Figure 8 presents the sequence of images that our wheelchair is entering using the mouth gesture mode.

As can be seen from the results presented in Table 4, the manual mode took less time and applied less number of input commands than the visual mode. The reason for the slowness of visual control mode is that: some mouth gesture control mode commands take a long time. For example, for executing the backward command, the user must open his/her mouth over 2 seconds. Also, the wrong commands that emerge in experiment 1 decrease with experiment 2 and experiment 3 while performing visual mode. The reason for the experiment 1 errors is that the user had not trained well to how performs the commands.

Table 4. Experiment results for both modes

\begin{tabular}{lllllll}
\hline & \multicolumn{3}{c}{ Manual mode } & \multicolumn{3}{c}{ Visual mode } \\
& Exp 1 & Exp 2 & Exp 3 & Exp 1 & Exp 2 & Exp 3 \\
\hline Traveling time (s) & 54 & 51 & 52 & 70 & 67 & 65 \\
Number of input commands & 18 & 16 & 18 & 23 & 21 & 21 \\
Number of wrong commands & 1 & 0 & 0 & 3 & 1 & 1 \\
\hline
\end{tabular}




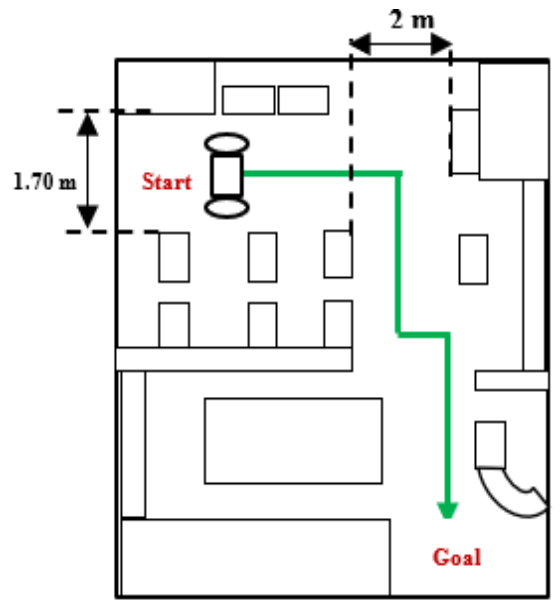

Figure 7. The route to follow in the laboratory environment

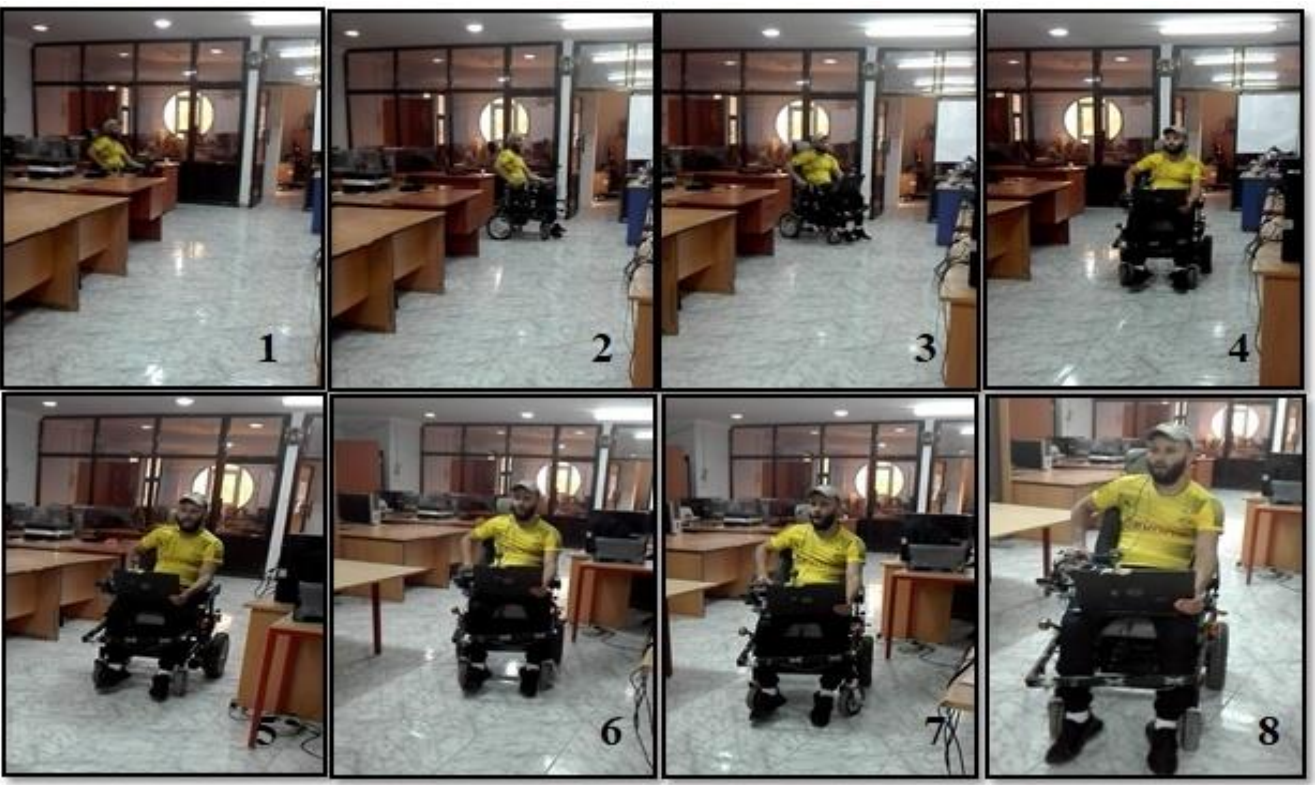

Figure 8. Experiments of mouth and tongue gestures control method

\section{CONCLUSION}

This paper represents the implementation of a new mouth and tongue gesture-based interface for controlling an IW. One of the main advantages of this system is that adaptable to various disabilities such as multiple sclerosis, quadriplegic, amputee's hand patients and old age people, also it is robust to a complex background. Another advantage is an accurate recognition of the user's intent with simple and few motions. The experimental results in the indoor environment show the success of the proposed control system. However, one can say that in order to reach these results through our system, a training session is recommended. As a perspective, the interest will be given to replace a laptop with a small single-board computers such as Raspberry Pi, to decrease the cost and produce a plug and play wheelchair control system, also to the development and designing of an intelligent wheelchair prototype with a multimodal interface in order to cover the needs of the disabled people whatever the level of their disability.

\section{REFERENCES}

[1] J. Leaman and H. M. La, "A Comprehensive Review of Smart Wheelchairs: Past, Present, and Future," in IEEE Transactions on Human-Machine Systems, vol. 47, no. 4, pp. 486-499, Aug. 2017, doi: 10.1109/THMS.2017.2706727. 
[2] C. Wahyufitriyani, S. Susmartini, and I. Priadythama, "Review of intelligent wheelchair technology control development in the last 12 years," 2016 2nd International Conference of Industrial, Mechanical, Electrical, and Chemical Engineering (ICIMECE), 2016, pp. 201-206, doi: 10.1109/ICIMECE.2016.7910458.

[3] W. Budiharto, and A. A. S.Gunawan, "Design and algorithm for electric wheelchair using voice recognition and joystick," ICIC Express Letters, vol. 12, no. 11, pp.1171-1175, 2018, doi:10.24507/icicel.12.11.1171.

[4] A. Škraba, R. Stojanović, A. Zupan, A. Koložvari, and D. Kofjač, "Speech-controlled cloud-based wheelchair platform for disabled persons," Microprocessors and Microsystems, vol. 39, no. 8, pp. 819-828, 2015, doi: 10.1016/j.micpro.2015.10.004.

[5] C. Aruna, A. Dhivya Parameswari, M. Malini, and G. Gopu, "Voice recognition and touch screen control based wheel chair for paraplegic persons," 2014 International Conference on Green Computing Communication and Electrical Engineering (ICGCCEE), 2014, pp. 1-5, doi: 10.1109/ICGCCEE.2014.6922215.

[6] A. S. Kundu, O. Mazumder, P. K. Lenka, and S. Bhaumik, "Hand Gesture Recognition Based Omnidirectional Wheelchair Control Using IMU and EMG Sensors," Journal of Intelligent \& Robotic Systems, vol. 91, no. 3, pp. 529-541, 2018, doi: 10.1007/s10846-017-0725-0.

[7] G. Jang, J. Kim, S. Lee, and Y. Choi, "EMG-Based Continuous Control Scheme With Simple Classifier for Electric-Powered Wheelchair," in IEEE Transactions on Industrial Electronics, vol. 63, no. 6, pp. 3695-3705, June 2016, doi: 10.1109/TIE.2016.2522385.

[8] R. Hardiansyah, A. Ainurrohmah, Y. Aniroh, and F. H. Tyas, "The electric wheelchair control using electromyography sensor of arm muscle," 2016 International Conference on Information \& Communication Technology and Systems (ICTS), 2016, pp. 129-134, doi: 10.1109/ICTS.2016.7910286.

[9] A. M. Choudhari, P. Porwal, V. Jonnalagedda, and F.Mériaudeau, "An Electrooculography based Human Machine Interface for wheelchair control," Biocybernetics and Biomedical Engineering, vol. 9, no. 3, pp. 673-685, 2019, doi: 10.1016/j.bbe.2019.04.002.

[10] Q. Huang et al., "An EOG-Based Human-Machine Interface for Wheelchair Control," in IEEE Transactions on Biomedical Engineering, vol. 65, no. 9, pp. 2023-2032, Sept. 2018, doi: 10.1109/TBME.2017.2732479.

[11] J. Tang, Y. Liu, D. Hu and Z. T. Zhou, "Towards BCI-actuated smart wheelchair system," BioMedical Engineering OnLine, vol. 17, no.1, 2018, doi: 10.1186/s12938-018-0545-x.

[12] G. Kucukyildiz, H. Ocak, S. Karakaya, and O. Sayli, "Design and Implementation of a Multi Sensor Based Brain Computer Interface for a Robotic Wheelchair," Journal of Intelligent \& Robotic Systems, vol. 87, pp. 247-263, 2017, doi: 10.1007/s10846-017-0477-x.

[13] R. Hassani, M. Boumehraz, M. Hamzi, and Z. Habba, "Gyro-Accelerometer based Control of an Intelligent Wheelchair," Journal of Applied Engineering Science \& Technology, vol. 4, no.1, pp.101-107, 2018.

[14] A. Al-Haddad, R. Sudirman, C. Omar, K. Y. Hui, and M. R. Jimin, "Wheelchair Motion Control Guide Using Eye Gaze and Blinks Based on PointBug Algorithm," 2012 Third International Conference on Intelligent Systems Modelling and Simulation, 2012, pp. 37-42, doi: 10.1109/ISMS.2012.23.

[15] M. A. Eid, N. Giakoumidis, and A. El Saddik, "A Novel Eye-Gaze-Controlled Wheelchair System for Navigating Unknown Environments: Case Study with a Person with ALS," in IEEE Access, vol. 4, pp. 558-573, 2016, doi: 10.1109/ACCESS.2016.2520093.

[16] R. Hassani, M. Boumehraz, and Z. Habba, "An Intelligent Wheelchair Control System based on Head Gesture Recognition," International Electrical and Computer Engineering Conference, 2015.

[17] Z. Hu, L. Li, Y. Luo, Y. Zhang, and X. Wei, "A novel intelligent wheelchair control approach based on head gesture recognition," 2010 International Conference on Computer Application and System Modeling (ICCASM 2010), 2010, pp. V6-159-V6-163, doi: 10.1109/ICCASM.2010.5619307.

[18] H. M. Qassim, A. K. Eesee, O. T. Osman, and M. S. Jarjees, "Controlling a motorized electric wheelchair based on face tilting," Bio-Algorithms and Med-Systems, vol. 15, no. 4, 2019, doi: 10.1515/bams-2019-0033.

[19] N. B. Viet, N. T. Hai, and N. V. Thuyen, "Hands-free control of an electric wheelchair using face behaviors," 2017 International Conference on System Science and Engineering (ICSSE), 2017, pp. 29-33, doi: 10.1109/ICSSE.2017.8030831.

[20] Y. Rabhi, M. Mrabet, and F. Fnaiech, "Intelligent control wheelchair using a new visual joystick," Journal of Healthcare Engineering, 2018, doi: 10.1155/2018/6083565.

[21] A. Zelinsky, "Learning OpenCV-Computer Vision with the OpenCV Library (Bradski, G.R. et al.; 2008)[On the Shelf]," in IEEE Robotics \& Automation Magazine, vol. 16, no. 3, pp. 100-100, September 2009, doi: 10.1109/MRA.2009.933612.

[22] P. Viola and M. J. Jones, "Robust real-time face detection," International Journal of Computer Vision, vol. 57, pp.137-154, 2004, doi: 10.1023/B:VISI.0000013087.49260.fb.

[23] A. H. Ahmad et al., "Real time face recognition of video surveillance system using haar cascade classifier," Indonesian Journal of Electrical Engineering and Computer Science, vol. 21, no. 3, pp. 1389-1399, 2021, doi: 10.11591/ijeecs.v21.i3.pp1389-1399.

[24] R. Brunelli, Template Matching Techniques in Computer Vision: Theory and Practice. New Jersey, USA: Wiley \& sons, 2009.

[25] Curtiss-Wright, JC2000 Multi Axis Contactless Joystick. Accessed: Sep. 03, 2020. [Online]. Available: www.cwindustrialgroup.com/getattachment/a6bb9db0-f4be-47c9-8e9d-e8931694969f/jc2000_brochure

[26] H. Dahou et al., "Design and Implementation Intelligent Adaptive Front-lighting System of Automobile using Digital Technology on Arduino board," International Journal of Electrical and Computer Engineering (IJECE), vol. 8, no. 1, pp. 521-529, 2018, doi: 10.11591/ijece.v8i1.pp521-529. 